IASSNS-HEP-98/88

hep-th/9811004

\title{
Brane Configurations for Branes at Conifolds
}

\author{
Angel M. Uranga \\ School of Natural Sciences, Institute for Advanced Study \\ Olden Lane, Princeton, NJ 08540
}

\begin{abstract}
We study the T duality between a set of type IIB D3 branes at non-orbifold threefold singularities, and type IIA configurations of D4 branes stretched between relatively rotated NS fivebranes. The four-dimensional $\mathcal{N}=1$ field theories on the D3 brane world-volume can be easily described using the IIA brane configuration. These models include families of chiral theories continuously connected to the theories appearing in brane box models (or D3 branes at orbifold singularities). We propose that phase transitions in the Kähler moduli space of the singularities are related to the crossing of rotated NS fivebranes in the T dual picture, and thus to Seiberg's duality in one of the gauge factors. We also comment on the inclusion of orientifold planes in the IIA brane picture.
\end{abstract}




\section{Introduction}

Configurations of NS fivebranes and D branes in string theory provide a useful and intuitive technique to study supersymmetric field theories in several dimensions (see [1] for a review with extensive references). A particularly interesting case is that of fourdimensional $\mathcal{N}=1$ gauge theories.

There are several approaches in the construction of these models 円. We briefly recall their basic ingredients. In [四], type IIB configurations of NS branes (of two kinds) and D5 branes were introduced to realize large families of chiral theories. These 'brane box models' have proved especially successful in the construction of $\mathcal{N}=1$ finite field theories [5].

A different construction, pioneered in [6], makes use of type IIA NS fivebranes (with relative rotations) and D4 branes stretching between them. These 'rotated brane configurations' generically realize non-chiral theories, but have been particularly useful in deriving large classes of Seiberg dual pairs [7], and in providing some exact results via their lifting to M-theory (see e.g. [8, 9, 10]).

Finally, it is possible to realize gauge field theories on the worldvolume of D3 brane probes of a certain spacetime background. The simplest example is placing D3 branes on a $\mathbf{C}^{3} / \Gamma$ singularity, with discrete $\Gamma \subset S U(3)$. The field theories, studied in [11 along the lines of [12], are generically chiral, and coincide with the theories obtained using certain brane box models. In [13] it was shown that both constructions are related by a T duality, which transforms the D5 branes into D3 branes, and the NS fivebranes into the singular geometry. These relations between different pictures of the same theory are always illuminating. For instance, in the case at hand, the finiteness results of [5] are closely related, using the singularity picture, to the conjectured AdS/CFT correspondence 114, 15, 16.

It is natural to seek the generalization to D3 brane probes in non-orbifold singularities. The simplest case, the conifold singularity, has been analyzed in [17], where a long distance field theory with the appropriate symmetries and physical behaviour was proposed, as arising on the D3 brane world-volume.

\footnotetext{
${ }^{1}$ Let us also mention the realizations in [2, 3, also in this spirit.
} 
Our purpose in the present paper is to explore the system of D3 branes at other nonorbifold singularities. The basic tool we exploit is to quotient the conifold variety $X$ by an appropriate discrete isometry group $\Gamma$. By determining the action of $\Gamma$ on the field theory, and keeping the dynamics of only the invariant states, the resulting gauge theory describes the D3 brane probes on the quotient $X / \Gamma$ (this type of 'orbifolding' of the field theory has been mainly studied for D3 branes on flat space [18]). Thus we construct large classes of chiral and non-chiral $\mathcal{N}=1$ field theories, with quartic superpotentials inherited from the conifold theory of [17].

We also show that these geometries are related to type IIA brane configurations of rotated NS fivebranes and D4 branes by a T duality along a direction transverse to the NS branes. This transformation, studied in 19 for the conifold, maps the D4 branes to D3 branes, and the NS fivebranes to the singular geometry. This relation nicely parallels the map between brane boxes and branes at orbifold singularities. We expect an interesting interplay of results from both pictures. Several results in this paper actually stem from T dual ways of looking at the same phenomenon: i) We argue that the continuation past infinite coupling in one of the gauge factors, realized as the crossing of two relatively rotated NS fivebranes in the type IIA brane picture, corresponds to transitions in the Kähler moduli space of the singular variety. ii) We describe explicitly the field theories of D3 branes at certain singularities which are partial smoothings of quotients of the conifold. The smoothing is mapped to the removal of certain NS fivebrane in the type IIA configuration, and to following a certain baryonic Higgs branch in the field theory. iii) We can generalize the constructions by including orientifold planes in the IIA brane picture. This corresponds to performing an orientifold projection in the singular geometry, whose direct analysis would be difficult without the guide of the $\mathrm{T}$ dual brane model.

The paper is organized as follows. In Section 2 we derive the T duality between D3 branes at the conifold geometry and the type IIA configuration of rotated NS branes and D4 branes. In Section 3 we consider singularities obtained as quotients of the conifold, and also find the T dual IIA brane models. We describe the D3 brane world-volume field theories, and perform some consistency checks. We also show that Kähler transitions in the singularity picture correspond to crossings of rotated NS fivebranes. In Section 4 we study D3 branes on partial resolutions of these singularities, describing the corresponding 
IIA pictures, and the resulting field theories. In Section 5 we consider quotients yielding chiral gauge theories. These models have Higgs branches along which the field theories correspond to finite brane box models (equivalently, D3 branes at $\mathbf{C}^{3} / \Gamma$ singularities). In Section 6 we discuss the inclusion of orientifold planes. Section 7 contains some final comments.

After this work was completed, we noticed reference [38], where a different approach to the problem of D3 branes at non-orbifold singularities is described. We expect further progress in the understanding of this system from the combination of different viewpoints.

\section{Brane configuration for the conifold}

Our starting point is a system of $N$ D3 branes sitting at the simplest non-orbifold threefold singularity, the conifold. The following analysis is similar to that of [19. The equation for the conifold

$$
x y=z w
$$

allows us to interpret the variety as a $\mathbf{C}^{*}$ fibration over the $\mathbf{C}^{2}$ parametrized by $z, w$. That is, for generic values of $z, w$, the variables $x, y$ describe a $\mathbf{C}^{*}$, since (2.1) can be used to relate $x$ and $y$ (as long as they are not zero or infinite). The fiber degenerates to two intersecting complex planes when the right hand side of (2.1) vanishes, i.e. along the (complex) curves $z=0$ and $w=0$ on the base.

Now we would like to perform a $\mathrm{T}$ duality along the $U(1)$ orbit in the $\mathbf{C}^{*}$ fiber (the orbit is composed of points which are related by $x \rightarrow \lambda x, y \rightarrow \lambda^{-1} y$, with $\lambda$ a complex number of unit modulus) \&. Following standard arguments [20] the degenerations in the fibration denote the presence of NS fivebranes in the T dual picture. In our case, we find two NS branes, which span the directions, say, 012345 and 012389 . We will denote them by NS and NS' branes, respectively. The T dual space is otherwise flat, and one direction transverse to the NS fivebranes, say 6, is compact.

\footnotetext{
${ }^{2}$ To be more precise, we would like to perform the $\mathrm{T}$ duality in a global model where this $S^{1}$ has constant radius far away from the degenerations.

${ }^{3}$ Notice however that the $\mathrm{T}$ duality we are performing differs from that considered in [20]
} 

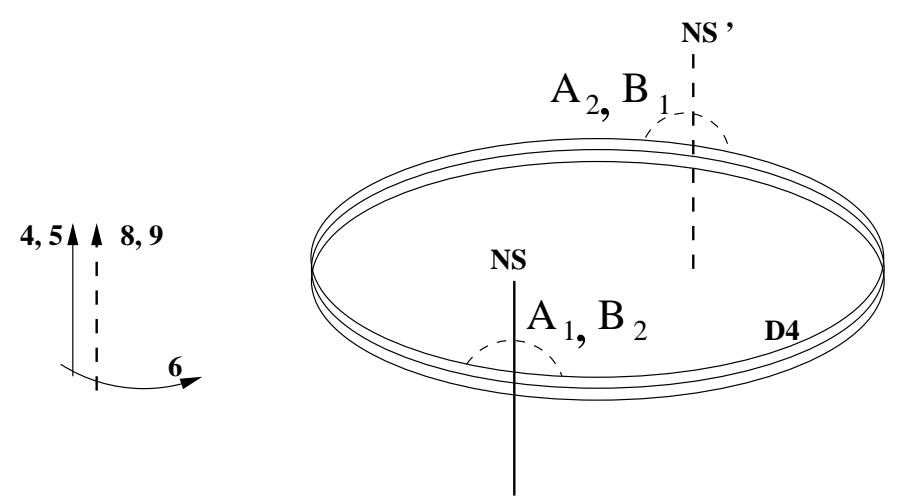

Figure 1: The type IIA brane configuration T dual to the system of D3 branes at the conifold singularity. In this and the following pictures, we represent the NS brane as a continuous vertical line, and the $\mathrm{NS}^{\prime}$ brane as a discontinuous vertical line. Since they are oriented along different directions (45 vs. 89), the D4 branes cannot separate and there is no Coulomb branch. Nevertheless, for the sake of clarity, we have depicted the D4 branes as slightly separated. We also show the chiral fields arising from open strings (depicted as a discontinuous curve) stretching between the D4 branes.

Notice that the two NS fivebranes need not be located at the same position in $x^{6}$. Their distance in $x^{6}$ is determined by the period of the NS two-form over the collapsed two-cycle in the conifold, whose value is not specified by the geometry (2.1). So, generically the NS fivebranes do not intersect, and define two intervals in the circle parametrized by $x^{6}$.

Finally, the D3 branes located at the conifold point in the singularity picture are mapped to D4 branes wrapping $x^{6}$. The resulting picture is shown in Figure 1 .

Type IIA brane configurations involving D4, NS and NS' branes have been considered in the literature (see [1] for references) in the case of non-compact $x^{6}$. Those analyses allow to read off the spectrum of the four-dimensional $\mathcal{N}=1$ field theory in our compact $x^{6}$ case as well.

The gauge group is $S U(N)^{2} \times U(1)$ (throughout this paper we will often ignore these decoupled $U(1)$ factors), and there are chiral multiplets $A_{1}, A_{2}$ in the representation ( $\left.\square, \bar{\square}\right)$, and $B_{1}, B_{2}$ in the $(\bar{\square}, \square)$. The adjoint chiral multiplets $\Phi, \Phi^{\prime}$ that would be massless if the NS fivebranes were parallel $(\mathcal{N}=2$ supersymmetric case [21]) receive a mass due to their relative rotation [22], which softly breaks the supersymmetry down to $\mathcal{N}=1$. The 
superpotential is

$$
W=A_{1} \Phi B_{2}-A_{2} \Phi B_{1}-A_{1} \Phi^{\prime} B_{2}+A_{2} \Phi^{\prime} B_{1}+m \Phi^{2}-m \Phi^{\prime 2}
$$

The adjoint masses are opposite since the two intervals have opposite rotation angle between their left and right NS fivebranes.

After integrating out the massive adjoints, the superpotential reads f

$$
W \propto \operatorname{Tr}\left(A_{1} B_{1} A_{2} B_{2}\right)-\operatorname{Tr}\left(A_{1} B_{2} A_{2} B_{1}\right) \propto \epsilon^{i j} \epsilon^{k l} \operatorname{Tr}\left(A_{i} B_{k} A_{j} B_{l}\right)
$$

The field content mentioned above, and the superpotential (2.3) define the field theory proposed in [17] as arising on the world-volume of D3 branes at the conifold singularity. We have rederived the result by $\mathrm{T}$ dualizing the geometry to a more familiar brane configuration. Notice that the relation of the conifold theory to the softly broken $\mathcal{N}=2$ theory was already uncovered in [17].

The type IIA brane configuration has the disadvantage that the $S U(2)^{2}$ global symmetry of the field theory (under which the $A_{i}$ and $B_{i}$ fields transform in the $(\mathbf{2}, \mathbf{1}$ ) and $(\mathbf{1}, \mathbf{2})$ representations, respectively) is not manifest, whereas in the conifold picture they are realized geometrically 円. However, brane configurations of this type often provide interesting insights on many issues. For instance, the inclusion of additional flavours in the field theories is straightforward in the IIA brane configuration, whereas in the IIB $\mathrm{T}$ dual it corresponds to including D7 branes, which introduce unpleasant dilaton-axion variations. So we feel it is worth exploring other field theories that can be realized in the IIA framework.

In the following sections we study D3 branes on other singularities, in some cases related to the conifold by a quotient, and describe candidate $\mathrm{T}$ dual IIA brane configurations.

\footnotetext{
${ }^{4}$ Notice that the NS fivebranes are at right angles, so the adjoint mass is naively infinite. However, it is clear that the brane configuration corresponds to a field theory with a non-vanishing quartic interaction, since it must have a possible Higgssing to the $\mathcal{N}=4$ theory, where there is a cubic superpotential.

${ }^{5}$ The $U(1)_{R}$ R-symmetry is realized geometrically in the IIA configuration as well.
} 


\section{Quotient of the conifold (I)}

In this section we describe a quotient of the conifold, and the field theory arising from D3 branes located at such singularity. We also present the $\mathrm{T}$ dual brane configuration, and provide some consistency checks of our identification.

\subsection{Description}

The knowledge of the field theory on D3 branes at the conifold can be exploited to analyze other singularities which are not orbifolds of $\mathbf{C}^{3}$. Simple examples of such worse singularities can be obtained by taking quotients of the conifold by some discrete symmetry. The conifold variety has an isometry group $S U(2) \times S U(2) \times U(1)_{R}$ (under which $x, y, z, w$ transform in the $(\mathbf{2}, \mathbf{2})_{+1}$ representation). In order to preserve $\mathcal{N}=1$ supersymmetry, the discrete groups will be embedded in the $S U(2)^{2}$ part, leaving the $U(1)_{R}$ R-symmetry untouched.

We will not attempt a general classification of such quotients, but present a few examples. A simple case, whose analysis we perform in the present section, is the $\mathbf{Z}_{k}$ action generated by:

$$
\begin{aligned}
& x \rightarrow e^{2 \pi i / k} x \\
& y \rightarrow e^{-2 \pi i / k} y
\end{aligned}
$$

leaving $z$ and $w$ invariant. By introducing the invariant variables $x^{\prime}=x^{k}, y^{\prime}=y^{k}, t=x y$, the final variety is described by the equations $x^{\prime} y^{\prime}=t^{k}, t=z w$, or equivalently by the expression

$$
x^{\prime} y^{\prime}=z^{k} w^{k}
$$

This singularity, in the form $\left(z_{1}^{2}+z_{2}^{2}\right)^{k}+z_{3}^{2}+z_{4}^{2}=0$, has appeared in [20, 23].

The field theory on the D3 branes can be obtained by an orbifolding procedure 18 (see also [24] for a general recipe in a purely field theoretical context). It is easy to see that the geometric action (3.1) corresponds to the following action on the field theory chiral multiplets

$$
\theta: \quad A_{1} \rightarrow \theta A_{1}
$$




$$
\begin{aligned}
& A_{2} \rightarrow \theta^{-1} A_{2} \\
& B_{1} \rightarrow \theta B_{1} \\
& B_{2} \rightarrow \theta^{-1} B_{2}
\end{aligned}
$$

with $\theta=e^{\pi i / k}$. To show this, recall the existence of a maximal Higgs branch where the matrices $A_{1}, A_{2}, B_{1}, B_{2}$ are diagonal and thus commuting. The $i^{\text {th }}$ eigenvalues $a_{1}, a_{2}, b_{1}$, $b_{2}$, parametrize the position of the $i^{\text {th }}$ D3 brane on the conifold by the relation

$$
x=a_{1} b_{1} \quad ; \quad y=a_{2} b_{2} \quad ; \quad z=a_{1} b_{2} \quad ; \quad w=a_{2} b_{1} .
$$

The action (3.3) must be embedded in the gauge degrees of freedom, which amounts to choosing an action on the Chan-Paton factors of the D3 branes. Starting with a conifold theory with group $S U(M k) \times S U(M k)$, we choose these embeddings to be given by the matrices

$$
\begin{aligned}
& \gamma_{\theta}^{(1)}=\operatorname{diag}\left(\mathbf{1}_{M}, \theta^{2} \mathbf{1}_{M}, \ldots, \theta^{2 k-2} \mathbf{1}_{M}\right) \\
& \gamma_{\theta}^{(2)}=\operatorname{diag}\left(\theta \mathbf{1}_{M}, \theta^{3} \mathbf{1}_{M}, \ldots, \theta^{2 k-1} \mathbf{1}_{M}\right)
\end{aligned}
$$

acting on the fundamental representations of the first and second factor, respectively. Here $\mathbf{1}_{M}$ denotes the rank $M$ identity matrix. The generalization to other embeddings with different number of entries for different eigenvalues is straightforward. In this respect, we should mention that in the singularity picture it is not obvious whether all such embeddings are consistent, since consistency conditions manifest as cancellation of tadpoles and these are not easy to compute for spaces other than orbifolds of flat space. The T-dual brane picture we will construct below, however, shows explicitly that all choices are consistent, and correspond to putting different numbers of D4 branes in the different $x^{6}$ intervals (this is analogous to the $\mathcal{N}=2$ case in [28]).

Going back to our choice of Chan-Paton factors (3.5), it is a simple matter to perform the projection on the fields of the theory. Each of the factors in the original gauge group $S U(M k) \times S U(M k)^{\prime}$ splits into $k$ identical $S U(M)$ factors, so the final $\mathcal{N}=1$ vector

\footnotetext{
${ }^{6}$ The Higgs branch is obtained by taking all such possible distributions of branes and dividing by the permutation group.
} 
multiplets form the group [1]

$$
S U(M)^{k} \times S U(M)^{\prime k}=\prod_{i=1}^{k} S U(M)_{i} \times \prod_{j=1}^{k} S U(M)_{j}^{\prime}
$$

where we have introduced labels to distinguish the factors.

The different $\mathcal{N}=1$ chiral multiplets suffer different projections depending on their global and gauge quantum numbers. For instance, the field $A_{1}$, in the $(\square, \bar{\square})$ of the initial group, has a projection

$$
\gamma_{\theta}^{(1)} A_{1}\left(\gamma_{\theta}^{(2)}\right)^{-1}=\theta A_{1}
$$

There are $k$ surviving chiral multiplets transforming in the representation $\left(\square_{i+1}, \bar{\square}_{i}^{\prime}\right)$ of $U(M)_{i+1} \times U(M)_{i}^{\prime}$, for $i=1, \ldots, k$. We will denote these fields by $\left(A_{1}\right)_{i+1, i}$.

Analogously, we obtain $k$ fields $\left(A_{2}\right)_{i, i}$ in the $\left(\square_{i}, \bar{\square}_{i}^{\prime}\right)$, fields $\left(B_{1}\right)_{i, i}$ in the $\left(\square_{i}^{\prime}, \bar{\square}_{i}\right)$, and fields $\left(B_{2}\right)_{i, i+1}$ in the $\left(\square_{i}^{\prime}, \bar{\square}_{i+1}\right)$. Notice that all these models are non-chiral.

The superpotential of the resulting theory is

$$
W \propto \sum_{i=1}^{k}\left(A_{1}\right)_{i+1, i}\left(B_{1}\right)_{i, i}\left(A_{2}\right)_{i, i}\left(B_{2}\right)_{i, i+1}-\sum_{i=1}^{k}\left(A_{1}\right)_{i+1, i}\left(B_{2}\right)_{i, i+1}\left(A_{2}\right)_{i+1, i+1}\left(B_{1}\right)_{i+1, i+1} .
$$

We can try to recover this field theory by a brane configuration related to the singularity via the T duality we discussed in Section 2. In order to do that, we interpret the defining equation $x^{\prime} y^{\prime}=z^{k} w^{k}$ as a $\mathbf{C}^{*}$ fibration over the $\mathbf{C}^{2}$ parametrized by $z, w$. The fiber degenerates again at the two complex planes $z=0$, and $w=0$. However, in this case the degeneration is worse, and the $\mathrm{T}$ dual picture contains $k$ NS branes and $k \mathrm{NS}^{\prime}$ branes. After taking into account the possibility of having B-fields in the singularity picture, the positions of the NS fivebranes in the $\mathrm{T}$ dual compact coordinate $x^{6}$ may differ. Again, the D3 branes sitting at the singularity become D4 branes wrapping $x^{6}$. The resulting IIA brane configuration looks like Figure 3 ?

\footnotetext{
${ }^{7}$ As usual in four-dimensional theories, the $U(1)$ factors (save for the overall one) are not present in the low-energy theory 21].

${ }^{8}$ Notice that for A-type fields the first (resp. second) index refers to unprimed (resp. primed) gauge factors, whereas for B-type fields the first (second) index refers to primed (unprimed) representation. This notations is convenient to suggest the appropriate contractions in the superpotential couplings.

${ }^{9}$ The orbifold field theory above is reproduced by a type IIA brane configuration where NS and NS' branes are arranged in an alternating fashion. In subsection 3.2 we will consider the interpretation of other possible orderings
} 


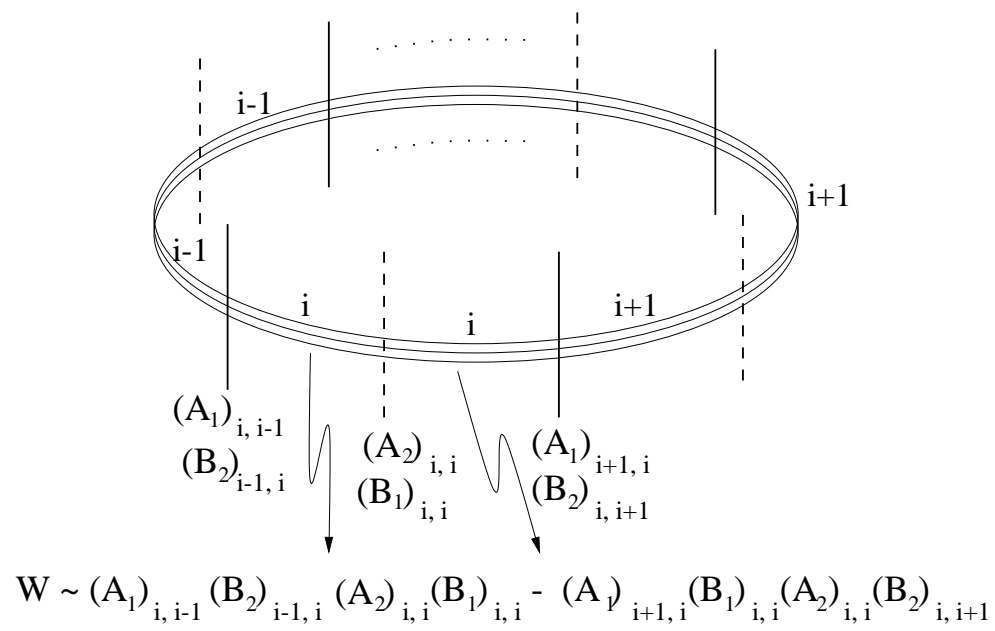

Figure 2: Brane configuration $\mathrm{T}$ dual to a system of D3 branes at the $x y=z^{k} w^{k}$ singularity. The model consists in a set of $k$ NS and $k \mathrm{NS}^{\prime}$ branes ordered along $x^{6}$ in an alternating fashion. The figure depicts the brane configuration in the vicinity of the $i^{\text {th }}$ pair of NS, NS' branes. We show the chiral fields in the bi-fundamental representations, and the superpotential quartic interactions mediated by the massive adjoints.

The four-dimensional field theory on the D-branes can be obtained by noticing it corresponds to softly breaking a $\mathcal{N}=2 S U(M)^{2 k}$ model down to $\mathcal{N}=1$ by appropriate adjoint masses. The gauge group is $\prod_{i=1}^{k} S U(M)_{i} \times \prod_{j=1}^{k} S U(M)_{j}^{\prime}$, where unprimed factors correspond to intervals with a NS brane on their left end, and primed factors to intervals with $\mathrm{NS}^{\prime}$ branes on their left end. It is easy to recognize the fields $\left(A_{1}\right)_{i, i-1},\left(B_{2}\right)_{i, i-1}$ as arising from open strings stretching between the D4 branes associated to $S U(M)_{i-1}^{\prime}$ and $S U(M)_{i}$; similarly, the fields $\left(A_{2}\right)_{i, i},\left(B_{1}\right)_{i, i}$ appear from open strings joining the D4 branes in the intervals corresponding to $S U(M)_{i}, S U(M)_{i}^{\prime}$. Finally, the superpotential is obtained after introducing the adjoint masses and integrating these fields out. This type of exercise has been performed e.g. in [25, 26], and leads to the appearance of quartic superpotentials. The result in our case yields the superpotential (3.8). Thus the proposed IIA brane configuration reproduces the orbifold field theory.

Before ending this subsection, we provide a few checks that support our identification. The first is the existence of mesonic Higgs branches in the field theory. These are manifest in the IIA brane configuration, where they amount to splitting the D4 branes 
at two adjacent NS branes (of the same kind), and moving the pieces in the direction 45, suspended between the NS branes. There are analogous Higgs branches in which pieces of D4 branes travel along 89, suspended between $\mathrm{NS}^{\prime}$ branes. An interesting hint is that the brane configuration localized near these traveling suspended D4 branes locally has $\mathcal{N}=2$ supersymmetry.

In the singularity picture, this phenomenon is accomplished by noticing that the singularity is not isolated. Actually, there is a curve of $\mathbf{C}^{2} / \mathbf{Z}_{k}$ singularities, parametrized by $w$, at $x=y=z=0$; and another similar curve, parametrized by $z$, at $x=y=w=0$. These curves arise from the set of points of the conifold which are fixed points under the $\mathbf{Z}_{k}$ action (3.1). The D3 branes at the origin can split into fractional branes [27] which can travel along these curves of singularities, but not away from them. This description of Higgs branches is identical to that in [28] for two-fold singularities and [13] for three-fold singularities. Along these branches, we have a set of D3 branes at a $\mathbf{Z}_{k}$ ALE singularity, a system with $\mathcal{N}=2$ supersymmetry.

Besides these mesonic branches, there are baryonic Higgs branches which, in the brane configuration, are realized as the removal of, say, one $\mathrm{NS}^{\prime}$ brane along $x^{7}$. In the field theory, it arises by giving diagonal vevs to the fields $\left(A_{2}\right)_{i, i},\left(B_{1}\right)_{i, i}$, which triggers the

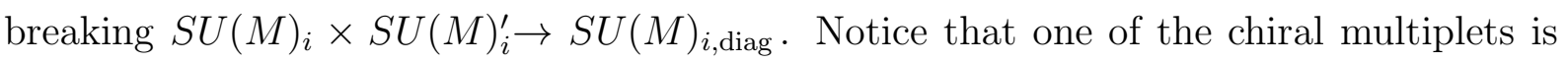
swallowed by the Higgs mechanism, whereas the other remains in the theory as a chiral multiplet in the adjoint. This remaining field parametrizes the possibility of moving the D4 branes along the two neighbouring NS branes. One such brane configuration is depicted in Figure 5. We will encounter again this type of brane configuration in Section 4, where we will see they are associated to partial resolutions of the singularity $x y=z^{k} w^{k}$.

These brane configurations can also be generalized by introducing D6 branes. This amounts to adding fundamental flavours in the field theory. In the $\mathrm{T}$ dual picture, this corresponds to the addition of D7 branes, and so the configuration is better described in terms of F-theory. We will not pursue these very interesting generalizations in the present paper. 


\subsection{Counting of marginal parameters}

In this subsection we are going to compute the number of exactly marginal operators in these field theories. Our motivation is to argue that the superpotential of the field theory we are studying can be defined, in analogy with [17], as a marginal deformation around a conformal point of the free theory. Also we show that most of the marginal couplings have a clear interpretation in the IIA brane construction.

From the field theory point of view, the number of marginal couplings can be determined using the techniques in [29], which were already exploited in [5] for a similar counting in brane box models (equivalently, D3 branes at orbifolds of $\mathbf{C}^{3}$ ). For notational clarity, let us momentarily denote the fields $\left(A_{1}\right)_{i+1, i},\left(B_{2}\right)_{i, i+1},\left(A_{2}\right)_{i, i}$ and $\left(B_{1}\right)_{i, i}$, by $\tilde{F}_{i}$, $F_{i}, \tilde{G}_{i}$ and $G_{i}$, respectively. The superpotential of the theory reads

$$
W=\sum_{i=1}^{k} \lambda_{i}^{(1)} F_{i} \tilde{F}_{i} G_{i} \tilde{G}_{i}+\sum_{i=1}^{k} \lambda_{i}^{(2)} \tilde{F}_{i} F_{i} \tilde{G}_{i+1} G_{i+1}
$$

where we have allowed for arbitrary superpotential couplings. The parameter space in the model is spanned by $2 k$ gauge couplings and $2 k$ superpotential couplings.

The conditions for a conformal theory can be found by using the exact beta functions for these parameters [30, 31]. For the gauge couplings of $S U(M)_{i}, S U(M)_{i}^{\prime}$, the vanishing of the beta function reads

$$
\begin{aligned}
& \beta_{g_{i}}=2+\gamma_{F_{i-1}}+\gamma_{\tilde{F}_{i-1}}+\gamma_{G_{i}}+\gamma_{\tilde{G}_{i}}=0 \\
& \beta_{g_{i}^{\prime}}=2+\gamma_{F_{i}}+\gamma_{\tilde{F}_{i}}+\gamma_{G_{i}}+\gamma_{\tilde{G}_{i}}=0
\end{aligned}
$$

where $\gamma_{X}$ is the anomalous dimension of the field $X$. For the superpotential couplings $\lambda_{i-1}^{(2)}, \lambda_{i}^{(1)}$, the vanishing of the beta functions are also given by the two equations (3.10), and do not provide independent constraints. Furthermore, there is one linear relation among the $2 k$ conditions (3.10), $\sum_{i} \beta_{g_{i}}=\sum_{i} \beta_{g_{i}^{\prime}}$. So we have a total of $2 k-1$ conditions on $4 k$ couplings, leading to a $(2 k+1)$-dimensional manifold of $\mathrm{RG}$ fixed points on the parameter space $\square$.

\footnotetext{
${ }^{10}$ The conifold case is special in that the two superpotential couplings are equal due to a global $S U(2) \times$ $S U(2)$ symmetry. This results in two marginal couplings 17] instead of the three our general counting indicates.
} 
The existence of these marginal couplings is directly inherited from the marginal superpotential in the conifold theory. Thus we can use their existence to define our field theory in the infrared in analogy with the argument in [17]. In the absence of superpotential, the field theory with the proposed matter content flows to a conformal theory. There it is possible to turn on the marginal couplings, and we recover the theory of interest.

The type IIA brane configuration provides a geometric realization of these marginal couplings. They correspond to the $k-1$ independent $x^{6}$ distances between NS branes of the same kind (adequately complexified by the shift in the type IIA RR $U(1)$ gauge field, or equivalently the positions of the NS branes in the eleventh dimension of M-theory [21]), the $k-1$ independent distances between NS ${ }^{\prime}$ branes, and the length of the $x^{6}$ coordinate (complexified to the complex structure of the torus parametrized by $x^{6}, x^{10}$ in M-theory). Another parameter corresponds to the relative positions of the sets of NS and NS' branes. Notice that a last marginal couplings seems to be not explicit in the brane construction.

\subsection{Kähler transitions vs. ordering of branes}

The proposal of a type IIA brane configuration $\mathrm{T}$ dual to the D3 branes at the singularity (3.2) poses a puzzle. There exist many different brane configurations containing $k$ NS branes and $k^{\prime} \mathrm{NS}^{\prime}$ branes, which differ in the ordering of the fivebranes along the coordinate $x^{6}$. Even though these are physically different, they all can be claimed, by the arguments in section 3.1, to be $\mathrm{T}$ dual to a set of D3 branes at the space (3.2). This singularity, however, does not seem to contain any degree of freedom corresponding to the multiple choices in the type IIA picture. The resolution of the puzzle consists precisely in a proper identification of these choices in the singularity picture. We will argue that the different orderings of branes correspond to different phases in the Kähler moduli space of the singularity

The singular variety (3.2) can be thought of as a smooth manifold in the limit in which a set of $\mathbb{P}_{1}$ 's shrinks to zero size. However, there are topologically different (but birrationally equivalent) smooth manifolds which can degenerate to the variety (3.2).

\footnotetext{
${ }^{11}$ I acknowledge R. von Unge for useful conversations on the contents of this section and on his work [45]. His comments encouraged me to correct several confusing statements present in a previous version.
} 
These manifolds are related by flop transitions (see e.g. [35, 36]). Thus there are different ways of resolving the singularity by restoring the finite size of different sets of shrunk $\mathbb{P}_{1}$ 's. In string theory the size of these cycles is complexified by the corresponding period (B-field) of the type IIB NS two-form. The moduli space spanned by these "complexified sizes' is known as 'complexified Kähler moduli space'.

Singularities in this moduli space arise at points where the size and B-field of a given cycle vanish. These loci are of complex codimension one and thus, paths interpolating between two points in moduli space can always avoid them. Even though this implies there are no true phase transitions in these interpolations, we will loosely use the term 'Kähler phase transition' to denote paths in moduli space that actually cross the singular point.

Our proposal is that for each possible configuration of NS and NS' branes on the $x^{6}$ circle there is a phase in the complexified Kähler moduli space of the T dual singularity. Also, the exchange of adjacent NS and NS' branes corresponds to Kähler transitions in which the B-field of a vanishing cycle changes sign. This can be understood as follows. Using the T duality map, it is easy to realize that when all NS and NS' branes are located at $x^{7}=0$ all the two-cycles in the dual variety have vanishing real size. However, the B-fields on these cycles generically do not vanish, and they encode the positions of the type IIA NS fivebranes along the $x^{6}$ circle. The process of crossing a NS and a NS brane $^{\prime}$ by moving them in $x^{6}$, while keeping them at $x^{7}=0$, is mapped to a transition where the B-field of the T dual two-cycle varies continuosly and changes sign in the process, while the real size of the cycle remains zero. The singular point in moduli space, where the twocycle has zero size and B-field, corresponds to the NS and NS' branes exactly intersecting. This point can be avoided by a slight $x^{7}$-separation of the NS and $\mathrm{NS}^{\prime}$ branes when the coincide in $x^{6}$, in the same way as the singularity in Kähler moduli space can be avoided by turning on a non-zero real size for the two-cycle when the B-field vanishes.

The natural context to study the geometry of Kähler moduli space is toric geometry. A detailed presentation of toric geometry is outside the scope of the present paper, so we will merely depict the relevant toric diagrams for convenience of the readers familiar with this formalism (we refer to [37], [35] for further details). Since the toric description is not essential for other sections, other readers are adviced not to worry about these alternative 
pictures.

It would be nice to have a precise match between the different orderings of fivebranes in $x^{6}$ and the different configurations of B-fields. This would require a precise description of the B-field moduli space, but the details of this characterization in toric geometry are unknown to us. On the other hand, toric geometry provides a simple description of the moduli space of real sizes of the two-cycles, where the problem reduces to determining the different triangulations of a polygon associated to the singularity. Thus each ordering of the fivebranes in the non-compact direction $x^{7}$ corresponds to a particular triangulation of the polygon.

We may expect an analogously simple structure for the moduli space of B-fields. Notice that it should correspond to the ordering of the fivebranes in the compact direction $x^{6}$, so the global considerations should be different. In what follows we describe a suggestive correspondence between the different orderings of fivebranes in $x^{6}$ with the different triangulations of a polygon, once we take into account certain 'compactification prescriptions' for the diagram, to be described below. Notice that in these figures the triangulations do not represent small resolutions of the singularity (all two-cycles have vanishing size when all fivebranes sit at $x^{7}=0$ ), but somehow represent different configurations of B-fields on the two-cycles. The precise meaning of the triangulations in this context is thus somewhat unclear.

In Figure 3 we have depicted the different triangulations of the diagram for the singularity $x y=z^{2} w^{2}$, along with the corresponding brane configuration ए2.

A subtlety in this identification is that some distributions of fivebranes only differ in a translation along $x^{6}$. The corresponding diagrams, on the other hand, look rather different. The explanation for this lack of symmetry is that the variety contains a noncompact $\mathbb{P}_{1}$, which can be understood in the toric diagram as arising from gluing together the two complex planes represented by vertical segments on the sides. This last $\mathbb{P}_{1}$ 'closes

\footnotetext{
${ }^{12}$ Other examples can be worked out similarly, the basic rules (for which I only have 'empirical' evidence) being as follows: i) Different toric diagrams related by a 'rotation' of their $\mathbb{P}_{1}$ 's as explained below correspond to the same brane configuration (up to an irrelevant rotation along $x^{6}$ ); ii) The existence, in the toric diagram, of triangles forming the diagram of $\mathbf{C} \times\left(\mathbf{C}^{2} / \mathbf{Z}_{k}\right)$ corresponds to the existence of $k$ parallel adjacent fivebranes, forming a $\mathcal{N}=2$ subsector.
} 

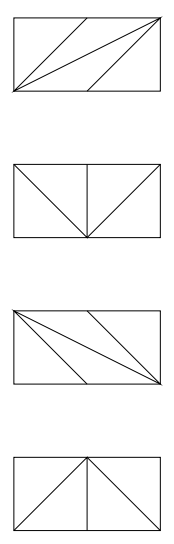
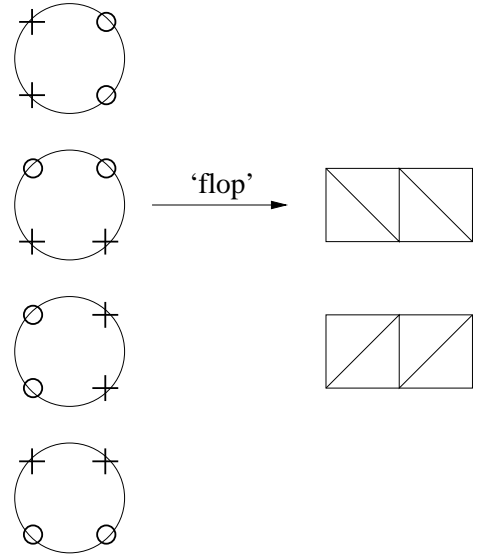
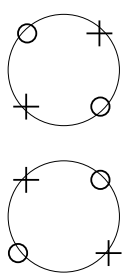

Figure 3: Comparison between the possible orderings of two NS branes and two NS' branes along $x^{6}$ and the triangulations of the diagram for the singularity $x y=z^{2} w^{2}$. The NS branes are represented by crosses, and the $\mathrm{NS}^{\prime}$ branes as circles, sitting at points in a circle representing the $x^{6}$ direction. We also show one example of a transition, and the corresponding exchange of $\mathrm{NS}$ and $\mathrm{NS}^{\prime}$ branes in the $\mathrm{T}$ dual brane picture.

up' a chain of $\mathbb{P}_{1}$ 's (in analogy with the way the affine node of an extended $A_{n}$ Dynkin diagram closes up the line of nodes of the non-extended diagram). The translation in $x^{6}$ in the brane picture somehow transforms the 'affine' $\mathbb{P}_{1}$ into a regular one, and a regular $\mathbb{P}_{1}$ into the new 'affine' one. One such process is illustrated in Figure $\mathbb{1}$, which should also clarify the systematics of our correspondence in Figure 3. This figure also illustrates the 'compactification prescription' that we mentioned above.

It is nice to observe in the examples depicted in Figure 3 how the Kähler transitions correspond to exchanges of pairs NS-NS'. However, some exchanges of branes relate toric diagrams which look very different. Again, this is the case when the additional 'affine' $\mathbb{P}_{1}$ is the 'flopped' one.

Let us turn to the field theory interpretation of this transitions. From the analysis in [6], the exchange of $\mathrm{NS}$ and $\mathrm{NS}^{\prime}$ branes is usually interpreted as $\mathcal{N}=1$ duality [7] between the gauge theories described by the initial and final brane configurations. In our case, the gauge group contains many factors and the crossing should correspond to dualizing just one of them. Isolating this sector of the model, the 'electric' theory belongs to a family of theories with gauge group $S U\left(N_{c}\right), n$ flavours $Q, \tilde{Q}$, and $m$ flavours $Q^{\prime}, \tilde{Q}^{\prime}$ 


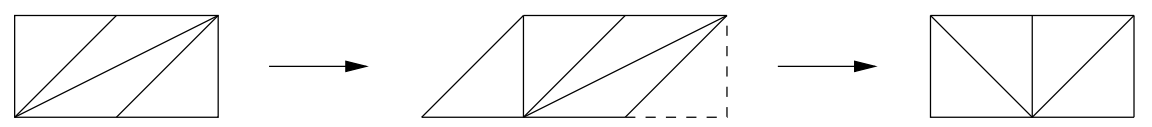

Figure 4: Intuitive argument showing that two triangulations corresponding to identical brane configurations differ by a 'rotation' among their $\mathbb{P}_{1}$ 's. In the first step, we 'cut' the lower left triangle piece of the toric diagram and 'glue' it on the other side. This amounts to making compact the previously non-compact $\mathbb{P}_{1}$, and making non-compact a previously compact one. The second step is a mere re-drawing of the same toric diagram.

all in the fundamental representation, and a quartic superpotential $W=(Q \tilde{Q})\left(Q^{\prime} \tilde{Q}^{\prime}\right)$. The 'magnetic' theory has group $S U\left(N_{c}-n-m\right), n$ flavours $q, \tilde{q}$ and $m$ flavours $q^{\prime}, \tilde{q}^{\prime}$, all in the fundamental, $n^{2}-1$ singlets mesons $M$, and $m^{2}-1$ singlet mesons $M^{\prime}$. The superpotential is $W=q M \tilde{q}+q^{\prime} M^{\prime} \tilde{q}^{\prime}+(q \tilde{q})\left(q^{\prime} \tilde{q}^{\prime}\right)$. The dual pair can be obtained from the model in [7] upon deforming it with the quartic superpotential, and has also been derived using brane configurations in [26]. In our type IIA construction, the transition of crossing the NS and $\mathrm{NS}^{\prime}$ branes reproduces this family of dual pairs. Different $m, n, N_{c}$ can be achieved by using different numbers of D4 branes on different intervals. In the singularity picture, they are mapped to fractional branes [28]. Observe that in our models the global symmetries of the field theory are gauged.

Notice that two theories related by brane crossing transitions form a dual pair if the field theories flow to strong coupling in the infrared. As intuitively argued in [2], in such case the NS fivebranes tend to come together and the two theories flow to the same configuration. According to this interpretation, the case $N_{c}=n=m$ in our particular example is quite subtle, since the corresponding field theory has a marginal coupling. If it indeed corresponds to the distance between the fivebranes in the brane construction, there is no apparent reason why the transition gives a dual pair, since in the IR this distance would remain non-zero. It would interesting to gain some insight on this issue. 


\section{Partial resolutions of the quotient of the conifold}

In this section our purpose is to analyze D3 branes on hypersurface singularities on $\mathbf{C}^{4}$ of the form

$$
x y=z^{n} w^{m}
$$

In general, these singularities are not quotients of the conifold, and thus the field theory on the D3 branes are not so straightforward to obtain. However, our T duality map still applies in analogy with previous sections, and one can use it to read off the field theory from the type IIA T dual brane configuration.

Following the usual argument, the $\mathrm{T}$ dual configuration contains $n$ NS branes and $m \mathrm{NS}^{\prime}$ branes, located at certain values in the $x^{6}$ coordinate. As we know, the specific ordering possibilities are in one-to-one correspondence with the different choices of Bfields of the singularity. Given one such type IIA brane configuration, the field theory is easily determined. The gauge group is $U(M)^{n+m}$. There are bifundamental fields in the $(\square, \bar{\square})+(\bar{\square}, \square)$ of adjacent gauge factors. Furthermore, there is an adjoint chiral multiplet whenever two NS fivebranes of the same kind are adjacent.

The superpotential can be determined by starting with an $\mathbf{Z}_{k}$ orbifold model as those studied in Section 3, with $k=\max (m, n)$, and removing the adequate number of NS or $\mathrm{NS}^{\prime}$ branes. In field theory language, this corresponds to going into the appropriate baryonic Higgs branches. The outcome of this exercise can be summarized in the following rules

- Whenever two relatively rotated NS fivebranes are adjacent, there is a quartic superpotential for the fields living at the ends (the interaction is mediated by the massive adjoint): $W= \pm F \tilde{F} G \tilde{G}$. The sign is taken positive (negative) if a NS $\left(\mathrm{NS}^{\prime}\right)$ brane lies at the left end of the interval.

- if two parallel NS fivebranes are adjacent, the superpotential is the usual $\mathcal{N}=2$ coupling between the adjoint and the chiral fields at the ends of the interval: $W=$ $F \Phi \tilde{F}-G \Phi \tilde{G}$.

Notice that the superpotential (3.8) can be obtained from the brane configuration Figure 2 by applying these rules. 
Just to provide an explicit example, we describe the specific case of the singularity $x y=z w^{2}$. In order to reach that model, we start with a $\mathbf{Z}_{2}$ quotient of the conifold, $x y=z^{2} w^{2}$, with group $S U(M)^{4}$, and matter fields

$\begin{array}{ccccc} & S U(M)_{1} & S U(M)_{2} & S U(M)_{3} & S U(M)_{4} \\ X_{12} & \square & \bar{\square} & 1 & 1 \\ X_{21} & \bar{\square} & \square & 1 & 1 \\ X_{23} & 1 & \square & \bar{\square} & 1 \\ X_{32} & 1 & \bar{\square} & \square & 1 \\ X_{34} & 1 & 1 & \square & \bar{\square} \\ X_{43} & 1 & 1 & \bar{\square} & \square \\ X_{41} & \bar{\square} & 1 & 1 & \square \\ X_{14} & \square & 1 & 1 & \bar{\square}\end{array}$

The superpotential is

$$
W=-X_{21} X_{12} X_{23} X_{32}+X_{32} X_{23} X_{34} X_{43}-X_{43} X_{34} X_{41} X_{14}+X_{14} X_{41} X_{12} X_{21}
$$

The removal of one NS brane corresponds to giving a diagonal vev to the field $X_{34}$. This breaks the gauge factors 3 and 4 to the diagonal combination, denoted 3 in the following. Thus the gauge group is $S U(M)_{1} \times S U(M)_{2} \times S U(M)_{3}$. The matter content is

$\begin{array}{cccc} & S U(M)_{1} & S U(M)_{2} & S U(M)_{3} \\ X_{12} & \square & \bar{\square} & 1 \\ X_{21} & \bar{\square} & \square & 1 \\ X_{23} & 1 & \square & \bar{\square} \\ X_{32} & 1 & \bar{\square} & \square \\ X_{31} & \bar{\square} & 1 & \square \\ X_{13} & \square & 1 & \bar{\square} \\ \Phi_{3} & 1 & 1 & \text { Adj. }\end{array}$

where the field $\Phi_{3}$ is the former $X_{34}$ that transforms in the adjoint of the diagonal group.

The brane configuration is depicted in Figure 5. The superpotential that follows from (4.2) reads

$$
W=-X_{21} X_{12} X_{23} X_{32}+X_{23} \Phi_{3} X_{32}-X_{13} \Phi_{3} X_{31}+X_{13} X_{31} X_{12} X_{21} .
$$




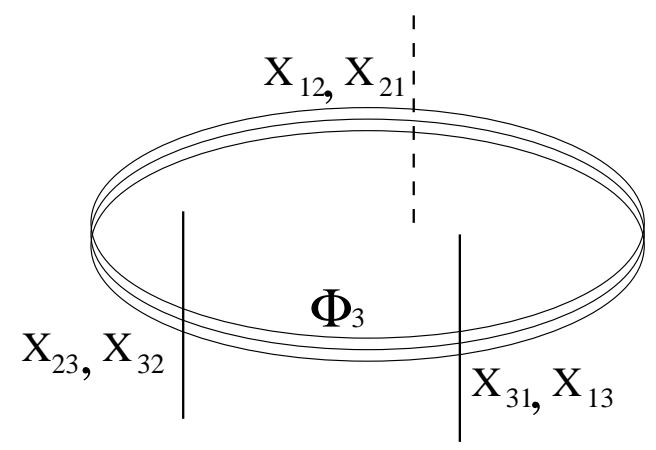

Figure 5: Brane configuration $\mathrm{T}$ dual to a system of D3 branes at the $x y=z w^{2}$ singularity.

We can easily check this also follows from our above rules. Other examples can be worked out analogously.

The theory of D3 branes at the singularity $x y=z w^{2}$ was also studied in [38]. Even though our derivation is different, the field theory we have proposed agrees with that in 38 .

Again, it is a nice exercise to match the Higgs branches of the field theory using both string theory descriptions: the IIA brane configuration, and the branes at singularities. For instance, as mentioned at the end of subsection 3.2, baryonic Higgs branches are realized by the removal of fivebranes in the brane configuration. It is clear that this allows to relate different theories in this class, so let us discuss how our rules to determine the superpotential take that into account.

Giving a diagonal vev to the field $X_{23}$ corresponds to removing one of the NS branes in the type IIA picture. The remaining configuration was proposed in section 2 as the T-dual to the conifold theory. This is neatly reproduced in the field theory, since the vev $<X_{23}>=v$ gives a mass to the $\Phi_{3}, X_{32}$. We integrate them out by using their equations of motion

$$
X_{32}=\frac{1}{v} \tilde{X}_{21} \tilde{X}_{12} \quad \Phi_{3}=X_{21} X_{12}
$$

Here $\tilde{X}_{12}, \tilde{X}_{21}$ are the former $X_{13}, X_{31}$. The final superpotential reads

$$
W=-X_{21} X_{12} \tilde{X}_{21} \tilde{X}_{12}+X_{21} \tilde{X}_{12} \tilde{X}_{21} X_{12}
$$

as corresponds to the conifold. 
a)

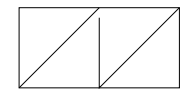

b)

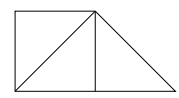

c)
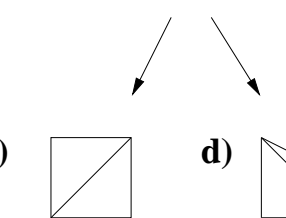

d)

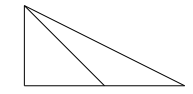

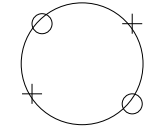

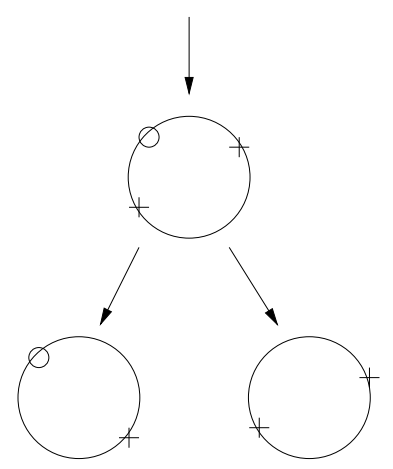

Figure 6: The toric diagrams corresponding to the singularities a) $x y=z^{2} w^{2}$, b) $x y=z w^{2}$, c) $x y=z w$, d) $x y=z^{2}$. We have also depicted the corresponding T dual IIA brane configurations, illustrating that the removal of NS fivebranes corresponds to the partial resolution of the singularity.

Instead, we can start with Figure 5, and follow the baryonic branch corresponding to removing the $\mathrm{NS}^{\prime}$ brane. This is accomplished by a diagonal vev for $X_{12}$. In this case no fields become massive, and the interactions (4.3) become the superpotential of an $\mathcal{N}=2$ $S U(M)^{2}$ elliptic model.

The removal of the fivebranes is clearly mapped, in the picture of branes at singularities, to the partial resolution of the singularity. This can be seen in the equation for the variety, or very pictorically, in the toric diagrams of these spaces. In Figure 6 we have depicted the toric diagrams of the singularities and how they are connected by the mentioned Higgs breakings.

\section{Quotients of the conifold (II): Chiral theories}

\section{$5.1 \quad \mathrm{Z}_{k} \times \mathrm{Z}_{l}$ orbifolds}

Even worse singularities can be analyzed by performing a further quotient to the theories studies in Section 3. For instance, we can consider quotients of the conifold by a $\mathbf{Z}_{k} \times \mathbf{Z}_{l}$ 
action, whose generators act as as

$$
\begin{aligned}
\alpha: \quad x & \rightarrow e^{2 \pi i / k} x \\
y & \rightarrow e^{-2 \pi i / k} y \quad z, w \text { invariant } \\
\beta: \quad z & \rightarrow e^{2 \pi i / l} z \\
w & \rightarrow e^{-2 \pi i / l} w \quad x, y \text { invariant. }
\end{aligned}
$$

It is straightforward to define invariant variables and check that the resulting manifold can be represented as the hypersurface

$$
x^{\prime l} y^{\prime l}=z^{\prime k} w^{\prime k}
$$

in $\mathbf{C}^{4}$. This singularity has appeared in [20], in the form $\left(z_{1}^{2}+z_{2}^{2}\right)^{k}+\left(z_{3}^{2}+z_{4}^{2}\right)^{l}=0$.

The action (5.2) has its counterpart on the fields $A_{i}, B_{i}$ of the conifold theory:

$$
\begin{array}{rlrl}
\theta: & A_{1} \rightarrow \theta A_{1} & \omega: & A_{1} \rightarrow \omega A_{1} \\
A_{2} & \rightarrow \theta^{-1} A_{2} & A_{2} \rightarrow \omega^{-1} A_{2} \\
B_{1} \rightarrow \theta B_{1} & B_{1} \rightarrow \omega^{-1} B_{1} \\
B_{2} \rightarrow \theta^{-1} B_{2} & & B_{2} \rightarrow \omega B_{2}
\end{array}
$$

where $\theta=e^{\pi i / k}, \omega=e^{\pi i / l}$.

Starting with a conifold theory with group $S U(M k l) \times S U(M k l)$, the embedding on the gauge degrees of freedom can be realized by the following Chan-Paton matrices:

$$
\begin{aligned}
& \gamma_{\theta}^{(1)}=\operatorname{diag}\left(\mathbf{1}_{M}, \mathbf{1}_{M} \ldots, \mathbf{1}_{M} ; \theta^{2} \mathbf{1}_{M}, \theta^{2} \mathbf{1}_{M} \ldots, \theta^{2} \mathbf{1}_{M} ; \ldots \ldots ; \theta^{2 k-2} \mathbf{1}_{M}, \theta^{2 k-2} \mathbf{1}_{M} \ldots, \theta^{2 k-2} \mathbf{1}_{M}\right) \\
& \gamma_{\theta}^{(2)}=\operatorname{diag}(\underbrace{\theta \mathbf{1}_{M}, \theta \mathbf{1}_{M} \ldots, \theta \mathbf{1}_{M}}_{l} ; \underbrace{\theta^{3} \mathbf{1}_{M}, \theta^{3} \mathbf{1}_{M} \ldots, \theta^{3} \mathbf{1}_{M}}_{l} ; \ldots, \underbrace{\theta^{2 k-1} \mathbf{1}_{M}, \theta^{2 k-1} \mathbf{1}_{M} \ldots, \theta^{2 k-1} \mathbf{1}_{M}}_{l}) \\
& \gamma_{\omega}^{(1)}=\operatorname{diag}\left(\mathbf{1}_{M}, \omega^{2} \mathbf{1}_{M} \ldots, \omega^{2 l-2} \mathbf{1}_{M} ; \mathbf{1}_{M}, \omega^{2} \mathbf{1}_{M} \ldots, \omega^{2 l-2} \mathbf{1}_{M} ; \ldots \ldots ; \mathbf{1}_{M}, \omega^{2} \mathbf{1}_{M} \ldots, \omega^{2 l-2} \mathbf{1}_{M}\right) \\
& \gamma_{\omega}^{(2)}=\operatorname{diag}\left(\omega \mathbf{1}_{M}, \omega^{3} \mathbf{1}_{M} \ldots, \omega^{2 l-1} \mathbf{1}_{M} ; \omega \mathbf{1}_{M}, \omega^{3} \mathbf{1}_{M} \ldots, \omega^{2 l-1} \mathbf{1}_{M} ; \ldots \ldots ; \omega \mathbf{1}_{M}, \omega^{3} \mathbf{1}_{M} \ldots, \omega^{2 l-1} \mathbf{1}_{M}\right)
\end{aligned}
$$

We stress that, contrary to the case in section 3.1, other choices of Chan-Paton factors may yield inconsistent theories. This is manifest since in this case the D3 brane world-volume theories are chiral and a generic choice of Chan-Paton matrices results in gauge anomalies in the field theory. The problem of determining the string consistency 
conditions in non-orbifold singularities seems rather complicated, and in the present work we will restrict to exploring the consistent choice shown above.

Using the projections induced by these actions, one can work out the field theory content. As expected, the gauge group in the orbifold models is

$$
\prod_{i=1}^{k} \prod_{j=1}^{l} S U(M)_{i, j} \times \prod_{i=1}^{k} \prod_{j=1}^{l} S U(M)_{i, j}^{\prime}
$$

The matter content is given by

$$
\begin{array}{ll}
\text { Field } & \text { Repr. } \\
\left(A_{1}\right)_{i+1, j+1 ; i, j} & \left(\square_{i+1, j+1}, \bar{\square}_{i, j}^{\prime}\right) \\
\left(A_{2}\right)_{i, j ; i, j} & \left(\square_{i, j}, \bar{\square}_{i, j}^{\prime}\right) \\
\left(B_{1}\right)_{i, j ; i, j+1} & \left(\square_{i, j}^{\prime}, \bar{\square}_{i, j+1}\right) \\
\left(B_{2}\right)_{i, j ; i+1, j} & \left(\square_{i, j}^{\prime}, \bar{\square}_{i+1, j}\right)
\end{array}
$$

Observe that these field theories are generically chiral. The superpotential is obtained by substitution of the surviving fields into the conifold superpotential. The outcome is the expression

$$
\begin{aligned}
W= & \sum_{i, j}\left(A_{1}\right)_{i+1, j+1 ; i, j}\left(B_{1}\right)_{i, j ; i, j+1}\left(A_{2}\right)_{i, j+1 ; i, j+1}\left(B_{2}\right)_{i, j+1 ; i+1, j+1}- \\
& -\sum_{i, j}\left(A_{1}\right)_{i+1, j+1 ; i, j}\left(B_{2}\right)_{i, j ; i+1, j}\left(A_{2}\right)_{i+1, j ; i+1, j}\left(B_{1}\right)_{i+1, j ; i+1, j+1}
\end{aligned}
$$

The space (5.3) has four curves of singularities radiating from the origin. Each is parametrized by either of the variables $x^{\prime}, y^{\prime}, z^{\prime}, w^{\prime}$, with the other variables equal to zero. It is possible to show that the Higgs branches corresponding to fractional D3 branes traveling along those curves actually exist in the field theory.

There are other interesting branches. For instance, giving a vev to all the fields $\left(A_{2}\right)_{i, j ; i, j}$, primed and unprimed factors with equal labels break to the diagonal combination. The final gauge group is $\prod_{i, j} S U(M)_{i, j}$. The surviving chiral multiplets are $\left(A_{1}\right)_{i, j ; i-1, j-1},\left(B_{1}\right)_{i, j ; i, j+1}$, and $\left(B_{2}\right)_{i, j ; i+1, j}$. The superpotential for these fields reads

$W \propto \sum_{i, j}\left[\left(A_{1}\right)_{i, j ; i-1, j-1}\left(B_{1}\right)_{i-1, j-1 ; i-1, j}\left(B_{2}\right)_{i-1, j ; i, j}-\left(A_{1}\right)_{i, j ; i-1, j-1}\left(B_{2}\right)_{i-1, j-1 ; i, j-1}\left(B_{1}\right)_{i, j-1 ; i, j}\right]$ 
This field theory is that appearing on D3 branes on a $\mathbf{C}^{3} /\left(\mathbf{Z}_{k} \times \mathbf{Z}_{l}\right)$ singularity [11], or equivalently [13] on a $k \times l$ brane box model with trivial identifications of the unit cell [5]. This fact will be recovered from the IIA brane configuration we are to propose as T-dual.

It is possible to construct a type IIA brane configuration such that the world-volume field theory on the D4 branes reproduces the theory just described. Consider the system of D4, NS and NS' branes T dual to a set of D3 branes on the $\mathbf{Z}_{k}$ quotient of the conifold, studied in Section 3. The models in this section are related to these theories by a further $\mathbf{Z}_{l}$ quotient. The proposal is to also quotient the type IIA configuration by the twist

$$
\begin{aligned}
x^{4}+i x^{5} & \rightarrow e^{2 \pi i / l}\left(x^{4}+i x^{5}\right) \\
x^{8}+i x^{9} & \rightarrow e^{2 \pi i / l}\left(x^{8}+i x^{9}\right)
\end{aligned}
$$

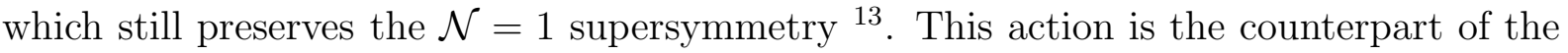
quotient by $\beta$ in (5.2). It must also be embedded on the Chan-Paton factors of the D4 branes. The final configuration provides a $\mathrm{T}$ dual realization of the field theory described above.

In order to check this claim, we can study the realization of some field theory Higgs branches in the branes setup. Obviously, some mesonic Higgs branches correspond to splitting the D4 branes between pairs of identical NS (resp. NS') branes, and moving them away along 45 (resp. 89). Observe that this phenomenon, when described in the covering space of the orbifold, requires the D4 branes to move along with their mirror images. These branches correspond, in the singularity picture, to the motion of fractional D3 branes along two of the curves of singularities mentioned above.

To see one of the remaining mesonic Higgs branches in the brane configuration, let us split the D4 branes in the intervals into fractional branes with respect to the quotient (5.7). The fractional branes of different intervals can recombine to form a fractional D4 brane going around the whole $x^{6}$ circle. These objects can move away from the NS fivebranes along $x^{7}$, but not away from the orbifold singularity. Finally, there is a last mesonic branch whose realization in the brane picture is not manifest.

Let us turn to some baryonic Higgs branches. It is possible to remove e.g. the $\mathrm{NS}^{\prime}$

\footnotetext{
${ }^{13}$ Brane configurations with orbifold quotients have been constructed in [3] (and in [39] with the inclusion of orientifold projections).
} 


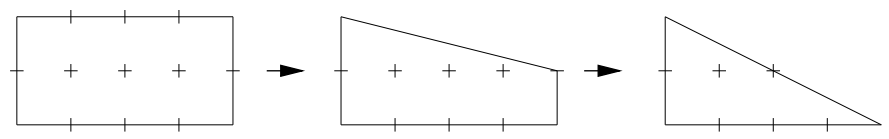

Figure 7: The first toric diagram represents the singularity $x^{2} y^{2}=z^{4} w^{4}$. The singularity can be partially resolved to the $\mathbf{C}^{3} /\left(\mathbf{Z}_{2} \times \mathbf{Z}_{4}\right)$ space. This process corresponds to the baryonic Higgs breaking mentioned in the main text.

branes along $x^{7}$. If all such branes are removed, the remaining brane configuration contains NS branes along 012345, D4 branes along 01236 and the $\mathbf{Z}_{l}$ orbifold quotient acting on 4589. By performing a T duality along 6, the NS branes become an ALE space, i.e. a $\mathbf{Z}_{l}$ orbifold quotient acting on 6789. The 'superposition' of both quotients results in a local $\mathbf{C}^{3} /\left(\mathbf{Z}_{k} \times \mathbf{Z}_{l}\right)$ singularity, at which D3 branes sit. Thus we recover the field theory Higgs branch we mentioned above.

It would be desirable to understand the meaning of possible transition in these more complicated singularities. Unfortunately, the IIA brane configuration we have constructed is not explicit enough to allow a direct comparison with the possible triangulations, as in the examples in section 3.3. We hope that a direct computation of the field theory using toric methods, along the lines of [38], will help in clarifying this issue.

In analogy with Section 3 we can now consider models with different numbers of NS and NS' branes. They provide T dual realization of the theory of D3 branes on singularities

$$
x^{l} y^{l}=z^{n} w^{m}
$$

The superpotentials for these theories are obtained by starting with the orbifold model and following the Higgs breakings in the field theory. In Figure 7 we illustrate how these breakings correspond to partial resolutions of the singular variety. The final model is reached from the initial one after a Higgssing along the baryonic branch mentioned above.

Even though the type IIA picture we have developed is somewhat complicated, it may prove useful to address certain issues. For instance, their lifting to M-theory seems to be quite tractable. However we will not pursue these points in the present work. 


\section{$5.2 \quad$ Isolated singularities}

In this section we consider other quotients of the conifold variety, which generically lead to isolated singularities (in contrast with the previous cases, where the orbifold action had fixed complex planes). This is achieved by using discrete groups under which $x, y, z$ and $w$ are non-invariant. We will show a concrete example, which has a simple realization in terms of the underlying variables $a_{i}, b_{i}$. The generator $\theta$ of the group acts as

$$
\begin{aligned}
& \theta: \quad a_{1} \rightarrow e^{2 \pi i \frac{1}{2 k}} a_{1} \quad x \rightarrow e^{2 \pi i(r+1) / k} x \\
& a_{2} \rightarrow e^{-2 \pi i \frac{1}{2 k}} a_{2} \quad y \rightarrow e^{-2 \pi i(r+1) / k} y \\
& b_{1} \rightarrow e^{2 \pi i \frac{2 r+1}{2 k}} b_{1} \quad z \rightarrow e^{-2 \pi i r / k} z \\
& b_{2} \rightarrow e^{-2 \pi i \frac{2 r+1}{2 k}} b_{2} \quad w \rightarrow e^{2 \pi i r / k} w
\end{aligned}
$$

Notice that, if $p_{1}=\operatorname{gcd}(\mathrm{k}, \mathrm{r}) \neq 1$, there are two complex planes (parametrized by $\left.z, w\right)$ of points fixed under a $\mathbf{Z}_{p_{1}}$ action, generated by $\theta^{k / p_{1}}$. Analogously, if $p_{2}=\operatorname{gcd}(k, r+1) \neq$ 1 , there are two complex planes (parametrized by $x, y$ ) of points fixed under a $\mathbf{Z}_{p_{2}}$ action, generated by $\theta^{k / p_{2}}$. For generic $k, r$, the singularity is isolated.

We choose to embed this action on the $S U(M k) \times S U(M k)^{\prime}$ gauge degrees of freedom though the matrices (3.5). The gauge group surviving the projection is $\prod_{i=1}^{k} S U(M)_{i} \times$ $\prod_{i=1}^{k} S U(M)_{i}^{\prime}$. The matter content is given by

$$
\begin{array}{ll}
\text { Field } & \text { Repr. } \\
\left(A_{1}\right)_{i+1, i} & \left(\square_{i+1}, \bar{\square}_{i}^{\prime}\right) \\
\left(A_{2}\right)_{i, i} & \left(\square_{i}, \bar{\square}_{i}^{\prime}\right) \\
\left(B_{1}\right)_{i+r, i} & \left(\square_{i+r}^{\prime}, \bar{\square}_{i}\right) \\
\left(B_{2}\right)_{i, i+r+1} & \left(\square_{i+r+1}^{\prime}, \bar{\square}_{i}\right)
\end{array}
$$

The field theories arising from this construction are thus generically chiral. The superpotential is

$$
\begin{aligned}
W= & \sum_{i=1}^{k}\left(A_{1}\right)_{i+1, i}\left(B_{1}\right)_{i, i-r}\left(A_{2}\right)_{i-r, i-r}\left(B_{2}\right)_{i-r, i+1}- \\
& \sum_{i=1}^{k}\left(A_{1}\right)_{i+1, i}\left(B_{2}\right)_{i, i+r+1}\left(A_{2}\right)_{i+r+1, i+r+1}\left(B_{1}\right)_{i+r+1, i+1} .
\end{aligned}
$$


For generic values of $k, r$ there are no mesonic branches, corresponding to the fact that there are no curves of singularities emanating from the origin. However, there is an interesting baryonic branch, which is obtained by giving diagonal vevs to, say, all the $\left(A_{2}\right)_{i, i}$ fields. The superpotential interaction for the remaining fields is

$$
W=\left(A_{1}\right)_{i+1, i}\left(B_{1}\right)_{i, i-r}\left(B_{2}\right)_{i-r, i+1}-\left(A_{1}\right)_{i+1, i}\left(B_{2}\right)_{i, i+r+1}\left(B_{1}\right)_{i+r+1, i+1}
$$

This field theory can also be obtained using a brane box model where the $k \times 1$ box unit cell has its vertical sides identified trivially, and its horizontal sides are identified up to a shift of $r$ boxes [5]. Equivalently [13], this theory arises from D3 branes on a $\mathbf{C}^{3} / \mathbf{Z}_{k}$ singularity, where the $\mathbf{Z}_{k}$ acts by multiplying the three complex planes by $e^{-2 \pi i / k}, e^{-2 \pi i r / k}$, $e^{2 \pi i(r+1) / k}$, respectively.

In principle, it would be possible to construct a type IIA brane configuration reproducing this field theory. The most systematic way would be to embed the action (5.9) in a $\mathbf{Z}_{n} \times \mathbf{Z}_{m}$ group of the type studied in section 5.1, in such a way that the subgroup can be recovered by quotienting by a suitable equivalence relation. The 'parent' singularity has a $\mathrm{T}$ dual IIA brane configuration which has been already described. The $\mathrm{T}$ brane configuration of the singularity of interest is obtained upon making the appropriate identifications in the 'parent' brane model. In particular these will amount to identifying fractional branes of different intervals. However, since this representation is rather involved, and its usefulness is doubtful, we will not extend on a general recipe.

\section{$6 \quad$ Introduction of orientifold planes}

The inclusion of orientifold planes in the type IIA brane picture is straightforward. For simplicity, we will only consider NS fivebranes at right angles. Even in this restricted context, there exist different patterns of orientifolded theories. We now turn to sketching them.

Starting with a brane configuration of the type studied in Section 3, we can include an orientifold four-plane, parallel to the D4 branes. These are $\mathcal{N}=1$ versions of the models studied in [40]. Recall that the charge of this orientifold changes sign whenever it crosses a NS fivebrane [41]. The general gauge group has a structure $S p \times S O \times S p \ldots \times S O$. 
Consistency requires the total number of NS fivebranes to be even. Once a distribution of NS and $\mathrm{NS}^{\prime}$ branes is chosen, the field theory content and superpotential can be read off from those of the non-orientifolded theory by simply projecting onto invariant fields. The effect of this projection on the gauge group has been already mentioned; also, after the projection, strings stretching between D4 branes of adjacent intervals give rise to only one chiral multiplet in the corresponding bi-fundamental representation. The surviving fields have a superpotential inherited from the non-orientifolded case.

Instead, we can introduce orientifold six-planes (O6 planes), extending along 0123789 [1. These are $\mathcal{N}=1$ versions of the models studied in 42. The orientifold projection inverts the $x^{6}$ coordinate, and so, working on the double cover, the distribution of NS fivebranes on the circle must be $\mathbf{Z}_{2}$ symmetric. There are two O6 planes, sitting at opposite position in the $x^{6}$ circle. The general pattern for the field theory gauge group is

$$
G_{1} \times S U \times \ldots \times S U \times G_{2}
$$

The $x^{6}$ intervals corresponding to the middle $S U$ factors are mapped to some 'mirror' intervals, and thus this sector of the theory is unrestricted, and has the structure of a non-orientifolded theory, namely the group factors are $S U$, and strings stretched between D4 branes yield two chiral fields, in the bi-fundamental and conjugate representations. The superpotential is also found using the rules in Section 3.

The 'end' sectors in the chain (6.1) correspond to the $x^{6}$ intervals closest to the O6 planes. There are several possibilities for this sector, depending on the distribution of fivebranes near the O6 plane. The basic building blocks have appeared in 443 피, and we schematically give them in the following table

\footnotetext{
${ }^{14}$ The configurations with $\mathrm{O} 6^{\prime}$ planes are obtained from those with $\mathrm{O} 6$ planes by exchange of the coordinates 45 and 89 , and so do not provide new models.

${ }^{15}$ The $\mathcal{N}=2$ building blocks were introduced in [44].
} 


$\begin{array}{ccccc}\text { Config. } & \text { Group } & \text { Matter } & \text { Superpot. } & \text { Comments } \\ N S-O 6^{+}-N S & S O & S & F S \tilde{F} & N=2 \\ N S-O 6^{-}-N S & S p & A & F A \tilde{F} & N=2 \\ N S^{\prime}-O 6^{+}-N S^{\prime} & S O & A & F A \tilde{F} & \\ N S^{\prime}-O 6^{-}-N S^{\prime} & S p & S & F S \tilde{F} & N=2 \\ N S-\left(N S, O 6^{+}\right)-N S & S U & S, \tilde{S}, X & S X \tilde{S}-F X \tilde{F} & N=2 \\ N S-\left(N S, O 6^{-}\right)-N S & S U & A, \tilde{A}, X & A X \tilde{A}-F X \tilde{F} & \\ N S^{\prime}-\left(N S, O 6^{+}\right)-N S^{\prime} & S U & S, \tilde{S} & S \tilde{S} F \tilde{F} & \\ N S^{\prime}-\left(N S, O 6^{-}\right)-N S^{\prime} & S U & A, \tilde{A} & A \tilde{A} F \tilde{F} & \text { Chiral } \\ N S-\left(N S^{\prime}, O 6\right)-N S & S U & A, \tilde{S}, 8 T & \tilde{S} T T+F \tilde{F} A \tilde{S} & \text { Chiral }\end{array}$

The notation is: $S(A)$ denote two-index symmetric (antisymmetric) representations, whereas $X$ and $T$ denote the adjoint and fundamental representations of $S U$, respectively. The field $F$ is a bi-fundamental field arising at the outer NS fivebranes in this sector. Tildes denote conjugate representations. When a NS fivebrane is stuck at the O6 plane, we write them both inside parentheses, otherwise the sequential order reproduces the ordering along $x^{6}$.

All these sectors can be introduced as endpoints in the chain (6.1). Once a given choice is made, it is straightforward to write down the field theory content and interactions.

A last type of configuration involving only NS fivebranes at right angles is possible by using NS fivebranes rotated $\pm 45^{\circ}$ in the 45,89 direction. We denote these as NS1 and NS2 branes, respectively. Notice that the orientifold plane maps NS1 to NS2 branes, since it inverts the coordinates 45 , while leaving 89 invariant. Thus, these type of branes cannot be stuck at the O6 planes. Again, the general gauge group has the structure (6.1), where the middle sector is that of a non-orientifolded theory. The endpoint sectors consist on a group factor $S O$ or $S p$ with no matter, save for bi-fundamentals. The superpotential for these last is quartic. If one allows for branes at arbitrary angles, it is possible to interpolate between this case and the one analyzed above.

It is clear that a large variety of models can be obtained if we also allow to quotient by $\mathbf{Z}_{k}$ actions in these brane configurations, in analogy with the models studied in Section 5. Moreover, it is also possible to include some orientifold projection acting along with this 
orbifold action. The scenarios can be complicated at wish, and it would be interesting to see what they can teach about orientifolding conifold (and generalized conifold) geometries in the $\mathrm{T}$ dual side. We leave all these points for future research.

\section{Final Comments}

We have proposed a T duality between D3 branes on some non-orbifold spaces and type IIA configurations of relatively rotated NS fivebranes and D4 branes. We expect a rich interplay between both realizations of the same field theories. Eventually, it may lead to an AdS realization of the diverse phenomena observed in lifting the IIA brane configuration to M-theory.

It would be desirable to have a better understanding on how the geometry of the singularity determines the field theory. We hope the examples we have developed allow to infer some rules in this respect. In particular, our correspondence between different triangulations of the toric diagram with different field theories points in this direction, and is worth of further exploration. These rules may eventually provide the key to understanding more general singularities. At this point, we should stress that we have restricted to brane configurations with fivebranes at right angles, and that relaxing this constraint leads to a larger family of T-dual singularities.

\section{Acknowledgements}

I am pleased to thank A. Klemm, and M.J. Strassler for useful discussions, and espe-

cially to K. Dasgupta for discussion and for sharing with me his related results with Sunil Mukhi. I am also grateful to M. González for advice and support. This work is supported by the Ramón Areces Foundation (Spain).

\section{References}

[1] A. Giveon and D. Kutasov, "Brane dynamics and gauge theory," hep-th/9802067.

[2] H. Ooguri and C. Vafa, "Geometry of $\mathrm{N}=1$ dualities in four-dimensions," Nucl. Phys. B500 (1997) 62, hep-th/9702180. 
[3] J. Lykken, E. Poppitz, and S. P. Trivedi, "Chiral gauge theories from D-branes," Phys. Lett. B416 (1998) 286, hep-th/9708134.

[4] A. Hanany and A. Zaffaroni, "On the realization of chiral four-dimensional gauge theories using branes," J. High Energy Phys. 05 (1998) 001, hep-th/9801134.

[5] A. Hanany, M. J. Strassler, and A. M. Uranga, "Finite theories and marginal operators on the brane," J. High Energy Phys. 06 (1998) 011, hep-th/9803086.

[6] S. Elitzur, A. Giveon, and D. Kutasov, "Branes and N=1 duality in string theory," Phys. Lett. B400 (1997) 269-274, hep-th/9702014.

[7] N. Seiberg, "Electric - magnetic duality in supersymmetric nonabelian gauge theories," Nucl. Phys. B435 (1995) 129-146, hep-th/9411149.

[8] E. Witten, "Branes and the dynamics of QCD," Nucl. Phys. B507 (1997) 658, hep-th/9706109.

[9] K. Hori, H. Ooguri, and Y. Oz, "Strong coupling dynamics of four-dimensional $\mathrm{N}=1$ gauge theories from $\mathrm{M}$ theory five-brane," Adv. Theor. Math. Phys. 1 (1998) $1-52$, hep-th/9706082.

[10] A. Brandhuber, N. Itzhaki, V. Kaplunovsky, J. Sonnenschein, and S. Yankielowicz, "Comments on the m theory approach to N=1 SQCD and brane dynamics," Phys. Lett. B410 (1997) 27, hep-th/9706127.

[11] M. R. Douglas, B. R. Greene, and D. R. Morrison, "Orbifold resolution by D-branes," Nucl. Phys. B506 (1997) 84, hep-th/9704151.

[12] M. R. Douglas and G. Moore, "D-branes, quivers, and ALE instantons," hep-th/9603167.

[13] A. Hanany and A. M. Uranga, "Brane boxes and branes on singularities," J. High Energy Phys. 05 (1998) 013, hep-th/9805139.

[14] J. Maldacena, "The large N limit of superconformal field theories and supergravity," hep-th/9711200. 
[15] S. S. Gubser, I. R. Klebanov, and A. M. Polyakov, "Gauge theory correlators from noncritical string theory," Phys. Lett. B428 (1998) 105, hep-th/9802109.

[16] E. Witten, "Anti-de Sitter space and holography," hep-th/9802150.

[17] I. R. Klebanov and E. Witten, "Superconformal field theory on three-branes at a Calabi-Yau singularity," hep-th/9807080.

[18] A. Lawrence, N. Nekrasov, and C. Vafa, "On conformal field theories in four-dimensions," hep-th/9803015.

[19] K. Dasgupta and S. Mukhi "Brane constructions, Conifolds and M theory", hep-th/9811139.

[20] M. Bershadsky, C. Vafa, and V. Sadov, "D strings on D manifolds," Nucl. Phys. B463 (1996) 398-414, hep-th/9510225.

[21] E. Witten, "Solutions of four-dimensional field theories via M theory," Nucl. Phys. B500 (1997) 3, hep-th/9703166.

[22] J. L. F. Barbon, "Rotated branes and N=1 duality," Phys. Lett. B402 (1997) 59-63, hep-th/9703051.

[23] H. Ooguri and C. Vafa, "Two-dimensional black hole and singularities of CY manifolds," Nucl. Phys. B463 (1996) 55-72, hep-th/9511164.

[24] M. Schmaltz, "Duality of nonsupersymmetric large N gauge theories," hep-th/9805218.

[25] J. H. Brodie and A. Hanany, "Type IIA superstrings, chiral symmetry, and N=1 4-d gauge theory dualities," Nucl. Phys. B506 (1997) 157, hep-th/9704043.

[26] O. Aharony and A. Hanany, "Branes, superpotentials and superconformal fixed points," Nucl. Phys. B504 (1997) 239, hep-th/9704170.

[27] M. R. Douglas, "Enhanced gauge symmetry in M(atrix) theory," J. High Energy Phys. 07 (1997) 004, hep-th/9612126. 
[28] A. Karch, D. Lüst, D. Smith, "Equivalence of geometric engineering and Hanany-Witten via fractional branes",Nucl. Phys. B533(1998)348, hep-th/9803232.

[29] R. G. Leigh and M. J. Strassler, "Exactly marginal operators and duality in four-dimensional N=1 supersymmetric gauge theory," Nucl. Phys. B447 (1995) 95-136, hep-th/9503121.

[30] M. A. Shifman and A. I. Vainshtein, "Solution of the anomaly puzzle in SUSY gauge theories and the Wilson operator expansion," Nucl. Phys. B277 (1986) 456.

[31] M. A. Shifman and A. I. Vainshtein, "On holomorphic dependence and infrared effects in supersymmetric gauge theories," Nucl. Phys. B359 (1991) 571-580.

[32] S. Kachru and E. Silverstein, "4-d conformal theories and strings on orbifolds," Phys. Rev. Lett. 80 (1998) 4855, hep-th/9802183.

[33] S. Gukov, "Comments on N=2 ads orbifolds," hep-th/9806180.

[34] S. Gukov and A. Kapustin, "New N=2 superconformal field theories from M / F theory orbifolds," hep-th/9808175.

[35] P. S. Aspinwall, B. R. Greene, and D. R. Morrison, "Calabi-Yau moduli space, mirror manifolds and space-time topology change in string theory," Nucl. Phys. B416 (1994) 414-480, hep-th/9309097.

[36] E. Witten, "Phases of $\mathrm{N}=2$ theories in two-dimensions," Nucl. Phys. B403 (1993) 159-222, hep-th/9301042.

[37] W. Fulton, "Introduction to toric varieties," Princeton Univ. Press (1993).

[38] D. R. Morrison and M. R. Plesser, "Nonspherical horizons, I," hep-th/9810201.

[39] J. Lykken, E. Poppitz, and S. P. Trivedi, "M(ore) on chiral gauge theories from D-branes," Nucl. Phys. B520 (1998) 51, hep-th/9712193.

[40] K. Landsteiner, E. Lopez, and D. A. Lowe, "N=2 supersymmetric gauge theories, branes and orientifolds," Nucl. Phys. B507 (1997) 197, hep-th/9705199. 
[41] N. Evans, C. V. Johnson, and A. D. Shapere, "Orientifolds, branes, and duality of 4-d gauge theories," Nucl. Phys. B505 (1997) 251, hep-th/9703210.

[42] A. M. Uranga, "Towards mass deformed $\mathrm{N}=4 \mathrm{SO}(\mathrm{n})$ and $\mathrm{Sp}(\mathrm{k})$ gauge theories from brane configurations," Nucl. Phys. B526 (1998) 241, hep-th/9803054.

[43] I. Brunner, A. Hanany, A. Karch, and D. Lust, "Brane dynamics and chiral nonchiral transitions," Nucl. Phys. B528 (1998) 197, hep-th/9801017.

[44] K. Landsteiner and E. Lopez, "New curves from branes," Nucl. Phys. B516 (1998) 273, hep-th/9708118.

[45] R. von Unge, "Branes at generalized conifolds and toric geometry", hep-th/9901091. 